\title{
Evalution of Surface Finish on Machining Of Mild Steel Using High Speed Steel Tool in Lathe with Normal Coolant (Or) Nano Material Added Coolant
}

\author{
T.Senthil Kumar ${ }^{1}$, G.Mahadevan ${ }^{2}$, T.R.Vikraman ${ }^{3}$ \\ Head Of Department, Department of Mechanical Engineering, BIT Campus, Anna University, Trichy, \\ Tamilnadu , India ${ }^{\text {, }}$ \\ Assistant Professor, Department of Mechanical Engineering, BIT Campus, Anna University, Trichy , \\ Tamilnadu, India ${ }^{2}$, \\ M.E. (Manufacturig Engg) student, Department of Mechanical Engineering, BIT Campus, Anna University, \\ Trichy, Tamilnadu, India ${ }^{3}$,
}

\begin{abstract}
Surface finish is one of the prime requirements of industrial machining. The purpose of this project is focused on the analysis of optimum cutting conditions to get lowest surface roughness in turning. This paper presents an experimental study to investigate the effects of cutting parameters like spindle speed, feed, depth of cut and nano material added type of coolant on surface finish on EN-8 by HSS M2 tool. The objective was to establish correlation between cutting speed, feed rate, depth of cut and type of coolant to optimize the turning conditions based on surface roughness. The study was conducted through RSM method with the help of Design Expert 8.0 software. We have used Servo cut of Indian oil or cool edge SL of Castrol used with water in a ratio of 1:20 as per manufacturer specification. The experiment conducted with 3 different speed, feed and DOC with out nano material ordinary coolant and with one nano material $\left(\mathrm{TiO}_{2}\right)$ included coolant for $\mathrm{L} 27$ method.

Key Words: Turning, Surface Roughness, Regression Surface Methodology(RSM), Sum Of Squares (SOS), Degree Of Freedom (DOF), surface roughness.
\end{abstract}

\section{Introduction}

Surface roughness has received serious attention for many years. It has formulated an important design feature in many situations such as parts subject to fatigue loads, precision fits, fastener holes, and aesthetic requirements. In addition to tolerances, surface roughness imposes one of the most critical constraints for the selection of machines and cutting parameters in process planning. Surface finish is the method of measuring the quality of a product and is an important parameter in machining process. It is one of the prime requirements of customers for machined parts. Productivity is also necessary to fulfill the customers demand. For this purpose quality of a product and productivity should be high. In addition to the surface finish quality is also an important characteristic in turning operation and high MRR is always desirable. Even in the occurrence of chatter or vibrations of the machine tool, defects in the structure of the work material, wear of tool, or irregularities of chip formation contribute to the surface damage in practice during machining.

1.1 Turning : Turning is a process, in which materials machined with the single point cutting tools which held stationary and work piece rotates. A large number of operations are required to produce the finished product, if some of the operations can be combined, or rough turning, heat treatment, and then grinding process. Surface roughness plays an important role as it influences the fatigue strength, wear rate, coefficient of friction, and corrosion resistance of the machined components. In actual practice, there are many factors which affect the surface roughness, i.e., tool variables, work piece hardness and cutting conditions. Theoretical turning, surface finish has been found to be influenced by a number of factors such as feed rate, cutting speed, tool nose radius and tool geometry, cutting time, work piece hardness, stability of the machine tool and the work piece set up. Surface roughness increases with increasing the feed rate but decreased with increasing the cutting speed and the depth of cut, respectively. The experiments were carried out using different feed rates $(0.05,0.10,0.15$ $\mathrm{mm} / \mathrm{rev})$ and different cutting speeds $(220,360,560 \mathrm{rpm})$ and different depth of cuts $(0.25,0.50,0.75 \mathrm{~mm})$ with (or) with out nano material $\left(\mathrm{TiO}_{2}\right)$ added fluid coolant. The turning operation is done on PSG A124 lathe (Fig $1)$. 


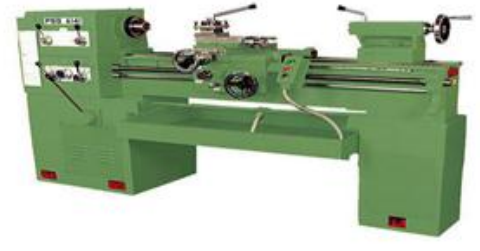

Fig 1

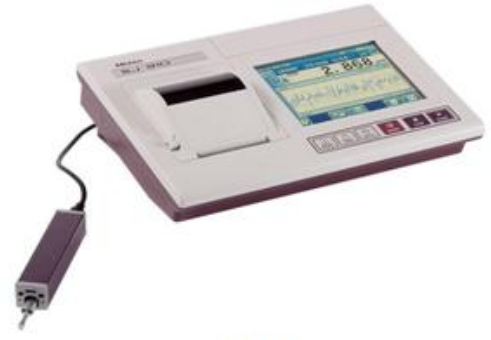

Fig 2

1.2. Measurement of Surface Roughness There are many ways to define surface roughness depending on its applications like $\mathrm{Ra}, \mathrm{Ry}, \mathrm{Rz}$, but roughness average $\mathrm{Ra}$ is widely used in industry for the mechanical components for indication of surface roughness, also known as arithmetic aver-age (AA) or centre line average (CLA) is the area between surface profile and centre line hence in this study $\mathrm{Ra}$ is used for indication of surface roughness $R a=1 / L \times(Y(x))$ Whereas $\mathrm{L}$ is the sample length, $\mathrm{Y}(\mathrm{x})$ is the profile along the direction $\mathrm{x}$. Surface roughness plays an important role in product quality. Developing an empirical model for the prediction of surface roughness in turning. The model considers the following working parameters feed, depth of cut, spindle speed, and nano material added coolant effect. Measured the surface roughness by Mitutoyo SJ310 (Fig 2).

\section{Experimental Work}

The experiment was conducted using one work piece material namely EN-8 with High Speed Steel tool and normal machine coolant. The tests were carried for a length of $100 \mathrm{~mm}$ and $\varnothing 28 \mathrm{~mm}$ in a PSG A124 lathe. The cutting parameters are shown in the Table 1. Three levels of cutting speed, three levels of feed and three levels of depth of cut were used and are shown in the Table 1. The different alloying elements present in a work piece and cutting tool are shown in the table $2 \& 3$.

\begin{tabular}{|l|l|l|l|}
\hline Cutting Parameters & $\begin{array}{l}\text { Level } \\
1\end{array}$ & $\begin{array}{l}\text { Level } \\
2\end{array}$ & $\begin{array}{l}\text { Level } \\
3\end{array}$ \\
\hline Speed (rpm) (A) & 220 & 360 & 560 \\
\hline Feed (mm) (B) & 0.05 & 0.10 & 0.15 \\
\hline Depth Of Cut (mm) (C) & 0.25 & 0.5 & 0.75 \\
\hline
\end{tabular}

Table 1

\subsection{HSS M2 Tool}

Shaping of M2 tool steels can be carried out using grinding methods. However, they have poor grinding capability and hence they are regarded as "medium" machinability tool steel under annealed conditions. The machinability of these steels is only $50 \%$ of that of the easily machinable $\mathrm{W}$ group or water hardening tool steels. Tool life HSS M2 is high-speed steel in tungsten-molybdenum series. The carbides in it are small and evenly distributed. It has high wear resistance. After heat treatment, its hardness is the same as T1, but its bending strength can reach $4700 \mathrm{M} \mathrm{Pa}$, and its toughness and thermo plasticity are higher than $\mathrm{T} 1$ by $50 \%$. It is usually used to manufacture a variety of tools, such as drill bits, taps and reamers. Its decarburization sensitivity is a little bit height.

\begin{tabular}{|l|l|l|l|l|l|}
\hline Grade & C & Cr & Mo & W & V \\
\hline M2 & 0.95 & 4.2 & 5.0 & 6.0 & 2.0 \\
\hline
\end{tabular}

\subsection{Work Piece EN 8}

Table 2

Mild steel EN8 size of D28mm $\times 100 \mathrm{~mm}$ rods. Mild steel is the most common form of steel because it's price is relatively low while it provides material properties that are acceptable for many applications, more so than iron. Low-carbon steel contains approximately $0.05-0.3 \%$ carbon. Mild steel has a relatively low tensile strength. Surface hardness can be increased through carburizing. It is often used when large quantities of steel are needed.

\begin{tabular}{|l|l|l|l|l|}
\hline $\mathrm{Cr}$ & $\mathrm{Mn}$ & $\mathrm{Si}$ & $\mathrm{P}$ & $\mathrm{S}$ \\
\hline 0.4 & 0.8 & 0.3 & 0.05 & 0.5 \\
\hline \multicolumn{5}{|c|}{ Table 3. }
\end{tabular}


Evalution of Surface Finish on Machining Of Mild Steel Using High Speed Steel Tool in Lathe ....

\section{Results And Discussion With Out Nano Fluid (Normal Coolant)}

\begin{tabular}{|c|c|c|c|c|c|c|c|}
\hline S1 no & Speed $(\mathrm{mm})$ & Feed $(\mathrm{mm})$ & $\begin{array}{l}\text { DOC } \\
(\mathrm{mm})\end{array}$ & $\begin{array}{l}\text { MRR (mm3/ } \\
\text { sec) }\end{array}$ & $\mathrm{Ra}(\mu \mathrm{m})$ & $\mathrm{Rq}(\mu \mathrm{m})$ & $\mathrm{Rz}(\mu \mathrm{m})$ \\
\hline 1 & 220 & 0.05 & 0.25 & 3.63 & 6.662 & 7.890 & 29.045 \\
\hline 2 & 220 & 0.05 & 0.50 & 7.34 & 5.330 & 6.368 & 25.452 \\
\hline 3 & 220 & 0.05 & 0.75 & 11.12 & 6.547 & 7.858 & 31.040 \\
\hline 4 & 220 & 0.10 & 0.25 & 7.26 & 4.856 & 6.141 & 23.180 \\
\hline 5 & 220 & 0.10 & 0.50 & 14.68 & 5.655 & 6.852 & 26.950 \\
\hline 6 & 220 & 0.10 & 0.75 & 22.25 & 5.141 & 6.423 & 24.379 \\
\hline 7 & 220 & 0.15 & 0.25 & 10.91 & 5.342 & 6.642 & 25.520 \\
\hline 8 & 220 & 0.15 & 0.50 & 22.03 & 6.902 & 8.447 & 34.781 \\
\hline 9 & 220 & 0.15 & 0.75 & 33.37 & 5.865 & 7.405 & 28.871 \\
\hline 10 & 360 & 0.05 & 0.25 & 5.95 & 4.241 & 5.137 & 22.041 \\
\hline 11 & 360 & 0.05 & 0.50 & 12.01 & 5.624 & 6.647 & 25.068 \\
\hline 12 & 360 & 0.05 & 0.75 & 18.20 & 5.131 & 6.314 & 28.121 \\
\hline 13 & 360 & 0.10 & 0.25 & 11.90 & 6.437 & 7.747 & 33.523 \\
\hline 14 & 360 & 0.10 & 0.50 & 24.02 & 5.821 & 7.414 & 32.937 \\
\hline 15 & 360 & 0.10 & 0.75 & 36.41 & 5.932 & 7.607 & 33.452 \\
\hline 16 & 360 & 0.15 & 0.25 & 17.85 & 5.769 & 7.340 & 32.540 \\
\hline 17 & 360 & 0.15 & 0.50 & 36.05 & 5.315 & 6.241 & 25.182 \\
\hline 18 & 360 & 0.15 & 0.75 & 54.61 & 4.450 & 5.415 & 23.947 \\
\hline 19 & 560 & 0.05 & 0.25 & 9.26 & 4.815 & 6.017 & 22.912 \\
\hline 20 & 560 & 0.05 & 0.50 & 18.68 & 5.234 & 6.214 & 23.851 \\
\hline 21 & 560 & 0.05 & 0.75 & 28.31 & 2.538 & 3.170 & 15.537 \\
\hline 22 & 560 & 0.10 & 0.25 & 18.51 & 4.831 & 5.771 & 23.051 \\
\hline 23 & 560 & 0.10 & 0.50 & 37.37 & 5.420 & 6.702 & 28.907 \\
\hline 24 & 560 & 0.10 & 0.75 & 56.63 & 5.314 & 6.301 & 25.326 \\
\hline 25 & 560 & 0.15 & 0.25 & 27.77 & 4.875 & 6.241 & 23.314 \\
\hline 26 & 560 & 0.15 & 0.50 & 56.08 & 4.307 & 5.221 & 22.992 \\
\hline 27 & 560 & 0.15 & 0.75 & 84.95 & 5.485 & 6.919 & 29.557 \\
\hline
\end{tabular}

Table 4.

3.1.1 Results And Discussion

Response 1 Ra ANOVA for Response Surface Quadratic Model Analysis of variance table [Partial sum of squares - Type III]

\begin{tabular}{|l|l|l|l|l|l|}
\hline Source & SOS & $\begin{array}{l}\text { DO } \\
\text { F }\end{array}$ & $\begin{array}{l}\text { Mean } \\
\text { Square }\end{array}$ & $\begin{array}{l}\text { Fo } \\
\text { Value }\end{array}$ & $\begin{array}{l}\text { P- Value } \\
\text { Prob>F }\end{array}$ \\
\hline $\begin{array}{l}\text { Model } \\
\text { Significan } \\
\text { t }\end{array}$ & 5.36 & 3 & 1.79 & 2.78 & 0.0640 \\
A - Speed & 5.15 & 1 & 5.15 & 8.01 & 0.0095 \\
\hline B - Feed & 0.16 & 1 & 0.16 & 0.25 & 0.6229 \\
\hline C - DOC & 0.048 & 1 & 0.048 & 0.075 & 0.7865 \\
\hline Residual & 14.78 & 23 & 0.64 & & \\
\hline Cor Total & 20.13 & 26 & & & \\
\hline
\end{tabular}

Table 5 Ra Calculation Table

Final equation of actual factors

$\mathrm{Ra}=6.45004-0.003129$ Speed +1.8833 Feed -0.20711 DOC

Response 2 Ry ANOVA for Response Surface 2FI ModelAnalysis of variance table [Partial sum of squares Type III]

\begin{tabular}{|l|l|l|l|l|l|}
\hline Source & SOS & $\begin{array}{l}\text { DO } \\
\text { F }\end{array}$ & $\begin{array}{l}\text { Mean } \\
\text { Square }\end{array}$ & $\begin{array}{l}\text { Fo } \\
\text { Value }\end{array}$ & $\begin{array}{l}\text { P- Value } \\
\text { Prob>F }\end{array}$ \\
\hline $\begin{array}{l}\text { Model } \\
\text { Significan } \\
\text { t }\end{array}$ & 8.44 & 3 & 2.81 & 3.01 & 0.0511 \\
\hline A - Speed & 7.52 & 1 & 7.52 & 8.03 & 0.0094 \\
\hline B - Feed & 0.85 & 1 & 0.85 & 0.90 & 0.3514 \\
\hline C - DOC & 0.075 & 1 & 0.075 & 0.080 & 0.7795 \\
\hline Residual & 21.54 & 23 & 0.94 & & \\
\hline Cor Total & 29.99 & 26 & & & \\
\hline
\end{tabular}


Table 6 Ry Calculation Table

Final equation of actual factors $\mathrm{Ry}=$ 7.68 $-\mathbf{0 . 0 0 3 7 8}$ Speed +4.339 Feed $-\mathbf{0 . 2 5 8 5} \mathrm{DOC}$

Response 3 Rz ANOVA for Response Surface Linear ModelAnalysis of variance table [Partial sum of squares - Type III]

\begin{tabular}{|l|l|l|l|l|l|}
\hline Source & SOS & $\begin{array}{l}\text { DO } \\
\text { F }\end{array}$ & $\begin{array}{l}\text { Mean } \\
\text { Square }\end{array}$ & $\begin{array}{l}\text { Fo } \\
\text { Value }\end{array}$ & $\begin{array}{l}\text { P- Value } \\
\text { Prob>F }\end{array}$ \\
\hline $\begin{array}{l}\text { Model } \\
\text { Significan } \\
\text { t }\end{array}$ & 112.23 & 3 & 37.41 & 2.12 & 0.1250 \\
A - Speed & 72.42 & 1 & 72.42 & 4.11 & 0.0544 \\
\hline B - Feed & 39.58 & 1 & 39.58 & 2.25 & 0.1476 \\
\hline C - DOC & 0.23 & 1 & 0.23 & 0.013 & 0.9093 \\
\hline Residual & 405.28 & 23 & 17.62 & & \\
\hline Cor Total & 517.51 & 26 & & & \\
\hline
\end{tabular}

Final equation of actual factors $R z=27.875-0.01173$ Speed +29.65 Feed $+\mathbf{0 . 4 5 5 7 8}$ DOC

In the next page graph between Speed and Feed for various feed rates and the difference between theoretical derived formula value and actual surface finish achieved is shown.

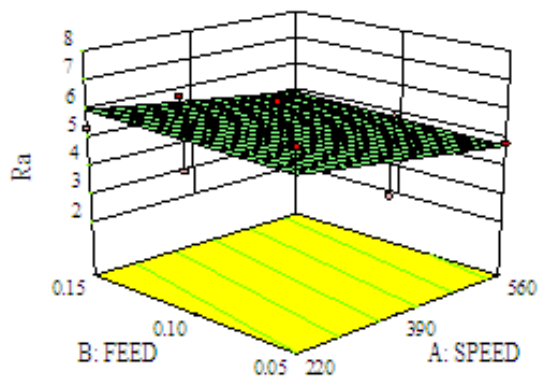

Graph 1: Ra for Speed with Feed of DOC 0.25

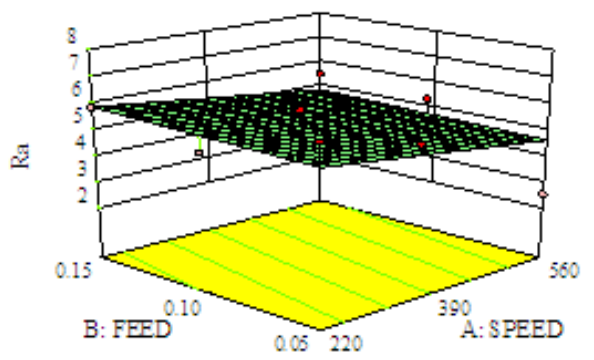

Graph 3:Ra for Speed with Feed of DOC 0.75

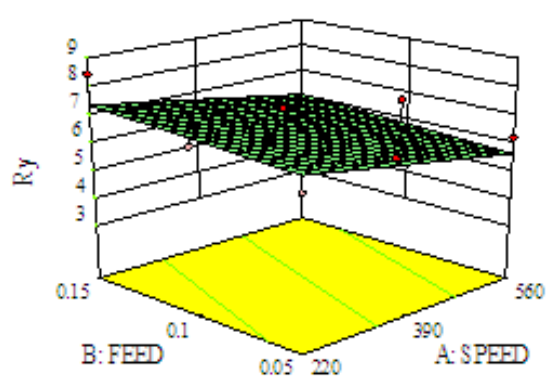

Graph 5:Ry for Speed with Feed of DOC 0.50

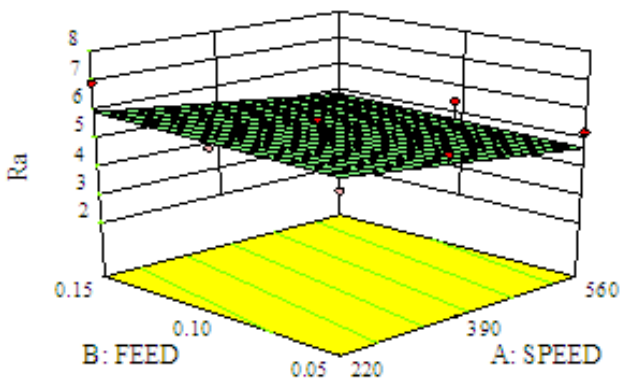

Graph 2: Ra for Speed with Feed of DOC 0.50

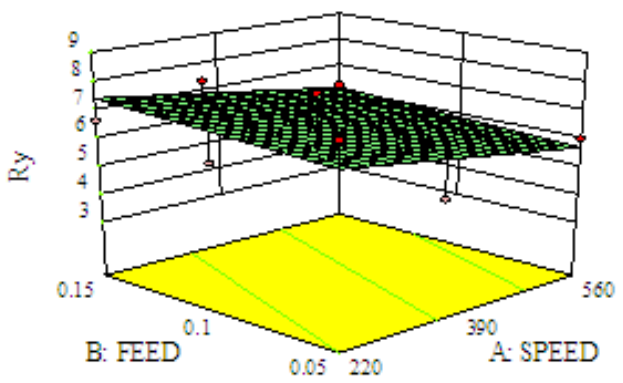

Graph 4:Ry for Speed with Feed of DOC 0.25

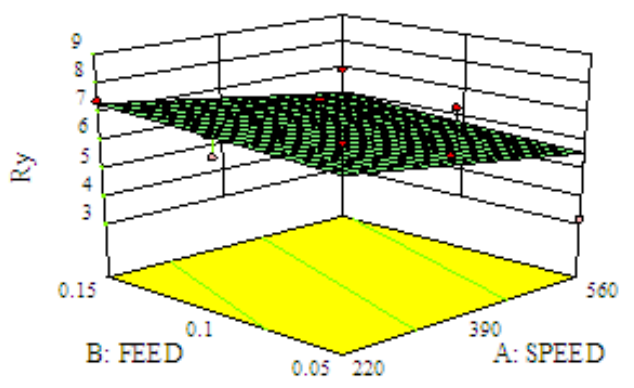

Graph 6:Ry for Speed with Feed of DOC 0.75 


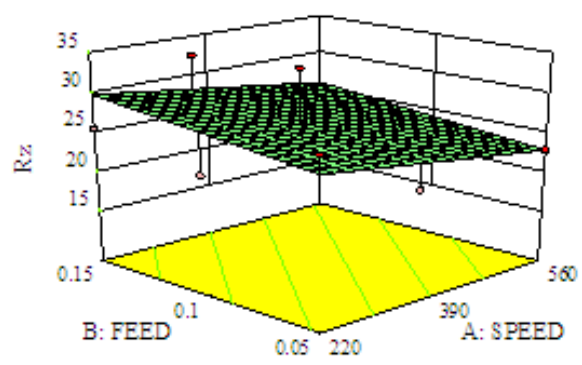

Graph 7:Rz for Speed with Feed of DOC 0.25

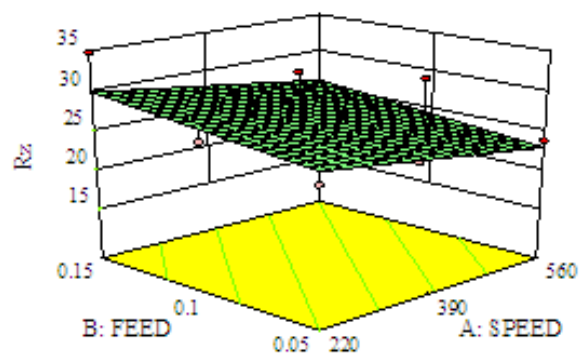

Graph 8:Rz for Speed with Feed of DOC 0.50

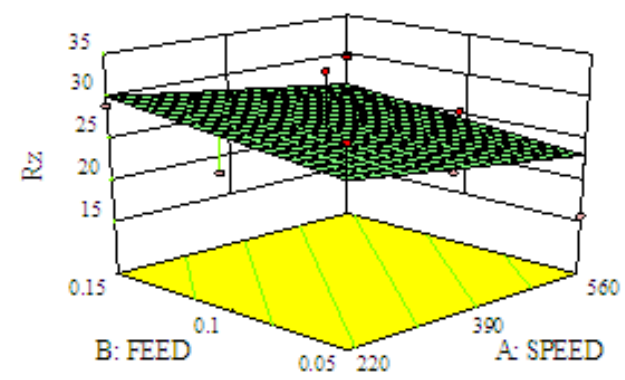

Graph 9:Rz for Speed with Feed of DOC 0.75
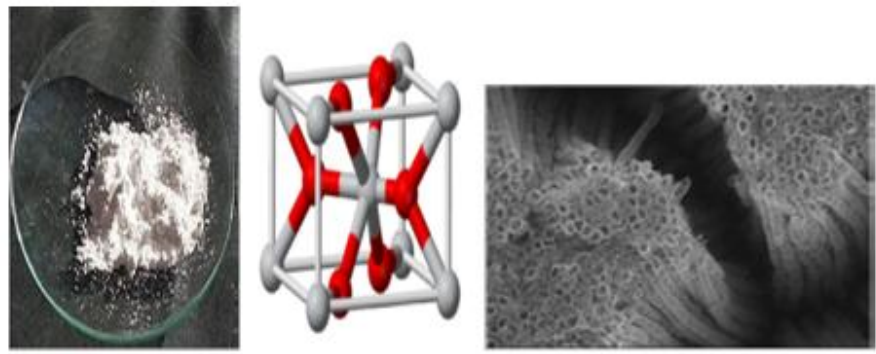

Figure 3. $\mathrm{TiO}_{2}$ Powder and nano tube construction

\subsubsection{Preparation of $\mathrm{TiO}_{2}$ nano} material added fluid

The $\mathrm{TiO}_{2}$ Nanoparticle is mixed with the conventional cutting fluid. The conventional cutting fluid is a mixture of soluble oil and water in the proportion of the $\mathrm{TiO}_{2}$ is mixed in the concentration of $0.1 \%$ with the cutting fluid. The nano cutting fluid is prepared for 3 litres. The mass of the $\mathrm{TiO}_{2}$ nanoparticle required for the preparation of nano fluid is calculated as follows

$$
\text { Density of } \mathrm{TiO}_{2}=4230 \mathrm{~kg} / \mathrm{m}^{3}, \quad \text { One litre }=\frac{1}{1000} \mathrm{~m}^{3}, \frac{\text { Mass }}{\text { Volume }}=\text { Density, }
$$

Mass $=$ Density $\times$ Volume, For 1 litre the mass of $\mathrm{Ti}_{2}$ required is Mass $=4230 \times \frac{1}{1000} \quad=4.23 \mathrm{~kg}$, At $0.1 \%$ concentration Mass $=4.23 \times \frac{0.1}{100} \times 1000=4.23 \mathrm{gm}$,

for 3 litres the mass of $\mathrm{Ti}_{2}$ is Mass $=4.23 \times 3$ $=12.69 \mathrm{gm}$

The mass of the $\mathrm{TiO}_{2}$ required for the preparation of the nanofluid is $12.69 \mathrm{gm}$. This Nano particle is mixed with the cutting fluid using "Ultrasonic Vibrator" in the Nano science laboratory. Then the Nano cutting fluid is used as the coolant the machining operation.

\begin{tabular}{|c|c|c|c|c|c|c|c|}
\hline S1 no & Speed $(\mathrm{mm})$ & Feed $(\mathrm{mm})$ & DOC $(\mathrm{mm})$ & $\begin{array}{c}\text { MRR }(\mathrm{mm} 3 / \\
\mathrm{sec})\end{array}$ & $\mathrm{Ra}(\mu \mathrm{m})$ & $\mathrm{Rq}(\mu \mathrm{m})$ & $\mathrm{Rz}(\mu \mathrm{m})$ \\
\hline 1 & 360 & 0.05 & 0.25 & 5.95 & 3.2255 & 3.568 & 21.2845 \\
\hline 2 & 360 & 0.10 & 0.25 & 11.90 & 4.021 & 4.4575 & 16.7535 \\
\hline 3 & 560 & 0.10 & 0.75 & 56.63 & 4.1245 & 4.3245 & 17.488 \\
\hline 4 & 560 & 0.05 & 0.50 & 18.68 & 2.1075 & 2.647 & 13.314 \\
\hline 5 & 360 & 0.10 & 0.50 & 24.02 & 4.261 & 4.471 & 17.2145 \\
\hline
\end{tabular}


Evalution of Surface Finish on Machining Of Mild Steel Using High Speed Steel Tool in Lathe ....

\begin{tabular}{|c|c|c|c|c|c|c|c|}
\hline 6 & 560 & 0.05 & 0.75 & 28.31 & 2.358 & 3.0255 & 14.2845 \\
\hline 7 & 560 & 0.10 & 0.50 & 37.37 & 3.9935 & 4.426 & 16.9265 \\
\hline 8 & 360 & 0.15 & 0.75 & 54.61 & 4.856 & 4.924 & 18.8425 \\
\hline 9 & 220 & 0.15 & 0.50 & 22.03 & 5.0125 & 5.654 & 22.8865 \\
\hline 10 & 560 & 0.05 & 0.25 & 9.26 & 2.2465 & 2.4285 & 12.6825 \\
\hline 11 & 360 & 0.15 & 0.50 & 36.05 & 4.769 & 5.1245 & 21.624 \\
\hline 12 & 560 & 0.15 & 0.75 & 84.95 & 4.594 & 4.8645 & 18.6142 \\
\hline 13 & 220 & 0.05 & 0.50 & 7.34 & 4.234 & 4.562 & 18.8785 \\
\hline 14 & 220 & 0.15 & 0.25 & 10.91 & 4.8665 & 5.1265 & 22.6475 \\
\hline 15 & 360 & 0.05 & 0.75 & 18.20 & 3.4565 & 5.8545 & 24.8755 \\
\hline 16 & 360 & 0.05 & 0.50 & 12.01 & 3.566 & 3.8945 & 17.426 \\
\hline 17 & 220 & 0.10 & 0.50 & 14.68 & 4.9645 & 5.214 & 21.642 \\
\hline 18 & 220 & 0.05 & 0.25 & 3.63 & 3.8465 & 3.9025 & 15.6465 \\
\hline 19 & 560 & 0.15 & 0.25 & 27.77 & 4.234 & 4.336 & 16.2855 \\
\hline 20 & 220 & 0.15 & 0.75 & 33.37 & 5.345 & 5.9855 & 23.1245 \\
\hline 21 & 220 & 0.10 & 0.25 & 7.26 & 4.6845 & 4.0245 & 16.0245 \\
\hline 22 & 220 & 0.10 & 0.75 & 22.25 & 5.126 & 5.3045 & 22.467 \\
\hline 23 & 220 & 0.05 & 0.75 & 11.12 & 4.6545 & 5.102 & 21.423 \\
\hline 24 & 360 & 0.10 & 0.75 & 36.41 & 4.3895 & 4.946 & 19.147 \\
\hline 25 & 360 & 0.15 & 0.25 & 17.85 & 4.3465 & 4.762 & 18.3425 \\
\hline 26 & 560 & 0.10 & 0.25 & 18.51 & 3.6325 & 3.942 & 16.984 \\
\hline 27 & 560 & 0.15 & 0.50 & 56.08 & 4.364 & 4.7365 & 18.4241 \\
\hline
\end{tabular}

Table 8 Experimental Readings

\subsubsection{RESULTS AND DISCUSSION}

Response 1 Ra ANOVA for Response Surface Quadratic Model Analysis of variance table [Partial sum of squares - Type III]

\begin{tabular}{|c|c|c|c|c|c|}
\hline Source & SOS & $\begin{array}{l}\text { DO } \\
\text { F }\end{array}$ & $\begin{array}{l}\text { Mean } \\
\text { Square }\end{array}$ & $\begin{array}{l}\text { Fo } \\
\text { Value }\end{array}$ & $\begin{array}{l}\text { P- Value } \\
\text { Prob }>F\end{array}$ \\
\hline $\begin{array}{l}\text { Model } \\
\text { Significa } \\
\text { nt }\end{array}$ & 18.74 & 9 & 2.08 & 86.82 & $<0.0001$ \\
\hline $\begin{array}{l}\text { A } \\
\text { Speed }\end{array}$ & 6.82 & 1 & 6.82 & 284.28 & $<0.0001$ \\
\hline B - Feed & 9.41 & 1 & 9.41 & 392.34 & $<0.0001$ \\
\hline $\mathrm{C}-\mathrm{DOC}$ & 0.77 & 1 & 1.37 & 57.24 & $<0.0001$ \\
\hline $\mathrm{AB}$ & 1.37 & 1 & 1.37 & 57.24 & $<0.0001$ \\
\hline $\mathrm{AC}$ & 0.045 & 1 & 0.045 & 1.87 & 0.1897 \\
\hline $\mathrm{BC}$ & $\begin{array}{l}3.23 x \\
10^{-3} \\
\end{array}$ & 1 & $\begin{array}{l}3.23 \times 1 \\
0^{-3}\end{array}$ & 0.13 & 0.7180 \\
\hline $\mathrm{A}^{2}$ & 0.12 & 1 & 0.12 & 5.02 & 0.0387 \\
\hline $\mathrm{B}^{2}$ & 0.74 & 1 & 0.74 & 30.76 & $<0.0001$ \\
\hline $\mathrm{C}^{2}$ & $\begin{array}{l}5.340 \times \\
10^{-6}\end{array}$ & 1 & $\begin{array}{l}5.340 \times \\
10^{-6}\end{array}$ & 0.22 & 0.6431 \\
\hline Residual & 0.41 & 17 & 0.024 & & \\
\hline $\begin{array}{l}\text { Cor } \\
\text { Total }\end{array}$ & 19.15 & 26 & & & \\
\hline
\end{tabular}

Table 9 Ra Calculation Table

Finalequationofactualfactors $R a=4.36881(0.010831 \times$ Speed $)+(26.45443 \times$ Feed $)+(1.73395 \times D O C)+(0.0$ $39594 \times$ Speed $\times$ Feed $)-$ $\left(1.42968 \times 10^{-3} \times\right.$ Speed $\times$ DOC $)+(1.3133 \times$ Feed $\times$ DOC $)+\left(5.08473 \times 10^{-}\right.$ ${ }^{6} \times$ Speed $\left.^{2}\right)-\left(140.2667 \times\right.$ Feed $\left.^{2}\right)-\left(0.47733 \times\right.$ DOC $\left.^{2}\right)$

Response 2 Ry ANOVA for Response Surface 2FI ModelAnalysis of variance table [Partial sum of squares Type III]

\begin{tabular}{|l|l|l|l|l|l|}
\hline Source & SOS & $\begin{array}{l}\text { DO } \\
\text { F }\end{array}$ & $\begin{array}{l}\text { Mean } \\
\text { Square }\end{array}$ & $\begin{array}{l}\text { Fo } \\
\text { Value }\end{array}$ & $\begin{array}{l}\text { P- Value } \\
\text { Prob }>\text { F }\end{array}$ \\
\hline Model Significant & 17.04 & 6 & 2.84 & 16.89 & $<0.0001$ \\
\hline A - Speed & 5.95 & 1 & 5.95 & 35.38 & $<0.0001$ \\
\hline B - Feed & 6.44 & 1 & 6.44 & 38.25 & 0.0003 \\
\hline C - DOC & 3.21 & 1 & 3.21 & 19.07 & 0.0491 \\
\hline AB & 0.74 & 1 & 0.74 & 4.39 & 0.2004 \\
\hline AC & 0.29 & 1 & 0.29 & 1.75 & 0.0898 \\
\hline BC & 0.53 & 1 & 0.53 & 3.18 & \\
\hline Residual & 3.36 & 20 & 0.17 & & \\
\hline Cor Total & 20.41 & 26 & \multicolumn{1}{|l}{} \\
\hline
\end{tabular}

Table 10 Ry Calculation Table 
Final equation of actual factors $R y=3.30956-\left(4.43364 \times 10^{-3} \times\right.$ Speed $)+(9.11066 \times$ Feed $)+(4.81313 \times D O C)+$ $(0.029035 \times$ Speed $\times$ Feed $)-\left(3.66963 \times 10^{-3} \times\right.$ Speed $\times$ DOC $)-(16.89 \times$ Feed $\times$ DOC $)$

Response 3 Rz ANOVA for Response Surface Linear ModelAnalysis of variance table [Partial sum of squares - Type III]

\begin{tabular}{|l|l|l|l|l|l|}
\hline Source & SOS & $\begin{array}{l}\text { DO } \\
\text { F }\end{array}$ & $\begin{array}{l}\text { Mean } \\
\text { Square }\end{array}$ & $\begin{array}{l}\text { Fo } \\
\text { Value }\end{array}$ & $\begin{array}{l}\text { P- Value } \\
\text { Prob>F }\end{array}$ \\
\hline $\begin{array}{l}\text { Model } \\
\text { Significan } \\
\text { t }\end{array}$ & 147.81 & 3 & 48.27 & 10.19 & 0.0002 \\
\hline A - Speed & 92.38 & 1 & 92.38 & 19.11 & 0.0002 \\
\hline B - Feed & 24.44 & 1 & 24.44 & 5.06 & 0.0344 \\
\hline C - DOC & 30.98 & 1 & 30.98 & 6.41 & 0.0186 \\
\hline Residual & 111.17 & 23 & 4.83 & & \\
\hline Cor Total & 258.98 & 26 & & & \\
\hline
\end{tabular}

Table $11 \mathrm{Rz}$ Calculation Table

Final equation of actual factors $R z=18.79626-(0.013257 \times$ Speed $)+$

$(23.30667 \times$ Feed $)+(5.24789 \times$ DOC $)$

In the next page graph between Speed and Feed for various feed rates and the difference between theoretical derived formula value and actual surface finish achieved is shown.

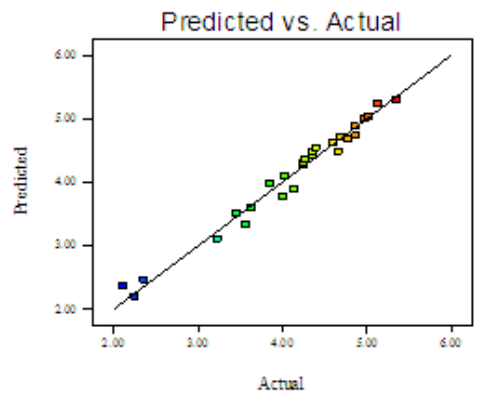

Graph 10: Ra actual and predicted

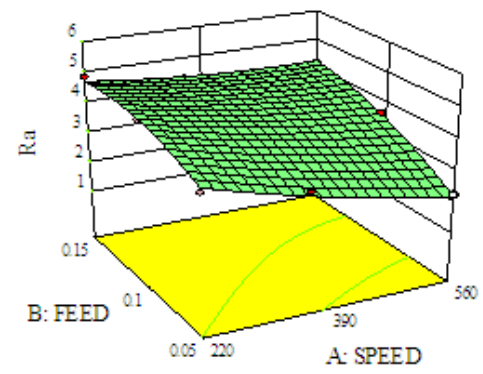

Graph 11: Ra for Speed with Feed of DOC 0.25

Feed of DOC 0.25

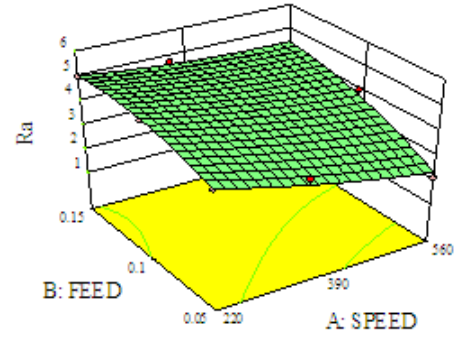

Graph 12: Ra Speed with Feed of DOC 0.50

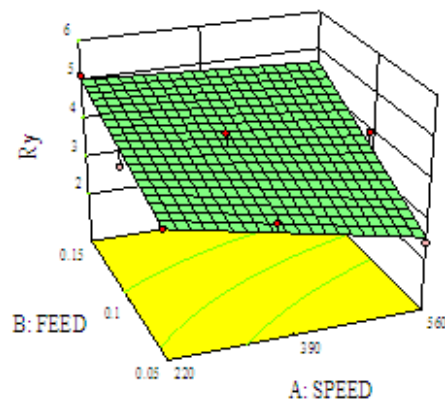

Graph 14: Ry for Speed with Feed of DOC 0.25

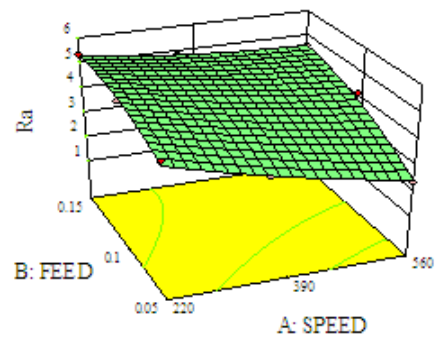

Graph 13: Ra Speed with Feed of DOC 0.75

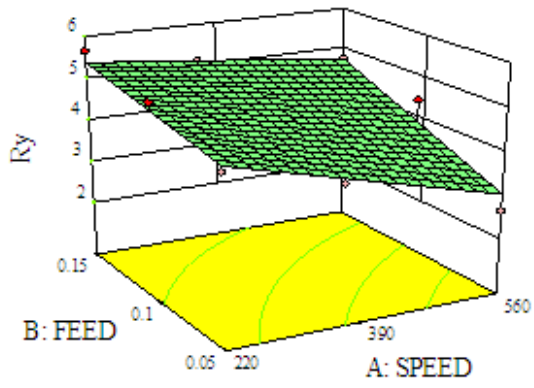

Graph 15 : Ry Speed with Feed of DOC 0.50 


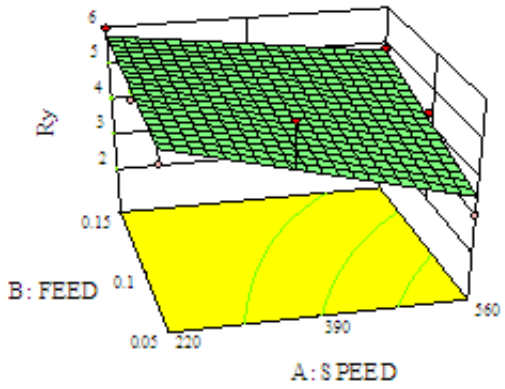

Graph 16: Ry Speed with Feed of DOC 0.75

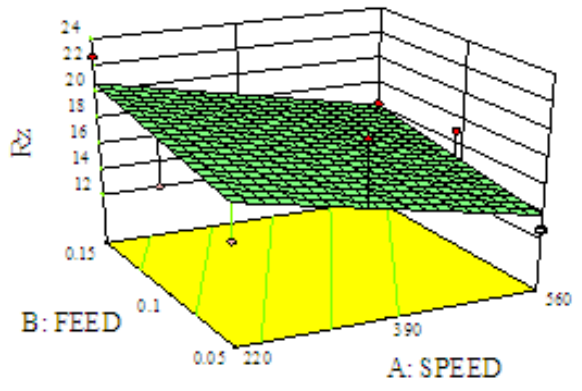

Graph 18: Rz Speed with Feed of DOC 0.50

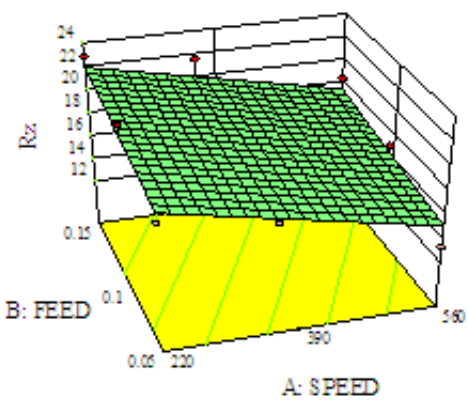

Graph 17: Rz Speed with Feed of DOC 0.25

3.3comparison Graph Between With And With Out Nano Material Added Coolant

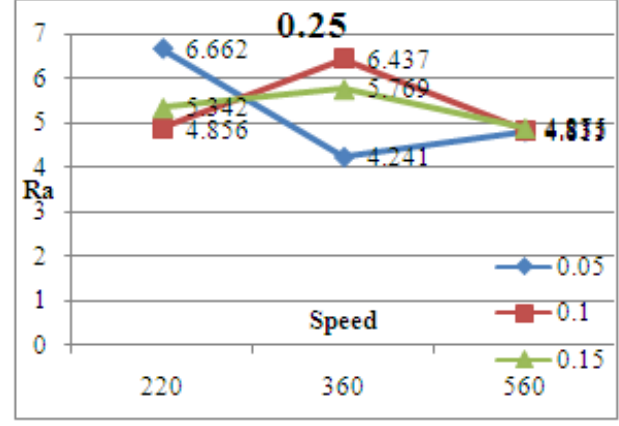

Graph: 20 Normal coolants

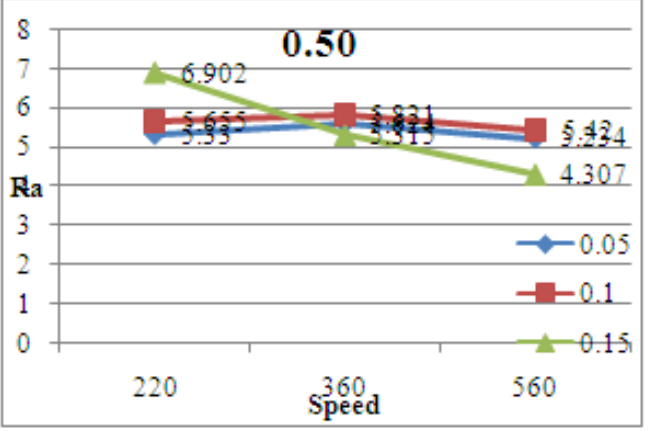

Graph: 21 Normal coolants

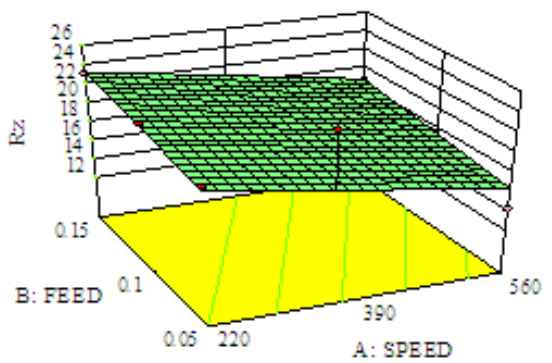

Graph 19: Rz Speed with Feed of DOC 0.75

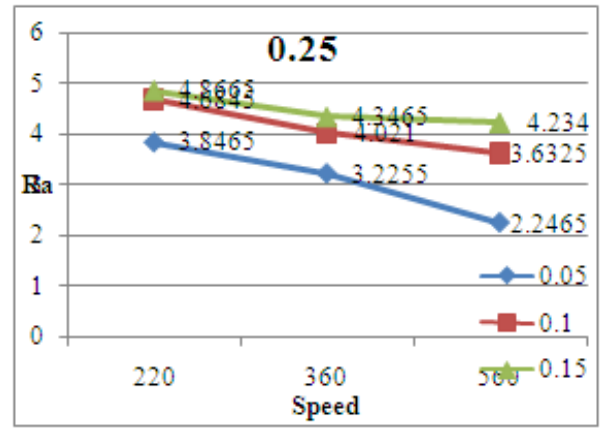

Graph: 21 Nano material added coolant

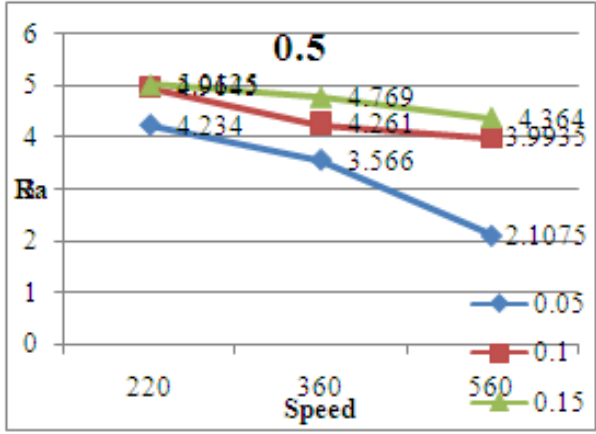

Graph: 22 Nano material added coolant 


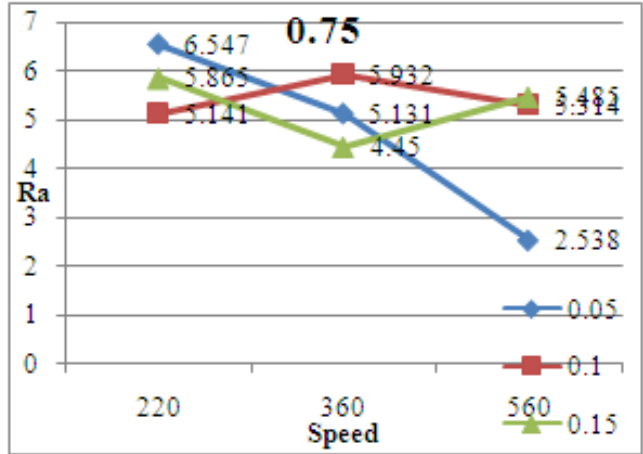

Graph: 23 Normal coolants

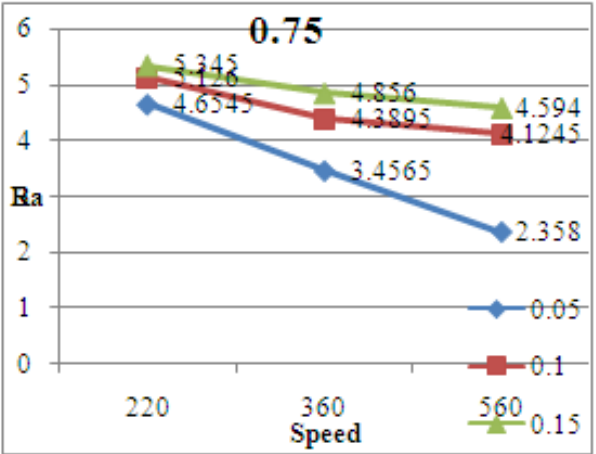

Graph: 24 Nano material added coolant

\section{Conclusion}

From the above with nomal coolant and with nano material added coolant both levels High Speed and Low feed rate gives minimum surface finish. When comparing coolant type the $\mathrm{TiO}_{2}$ added nano material coolant gives good level surface finish reading. From the ANOVA tabulations and calculation the parameters which influence the Surface Roughness are found. The factors and the levels that influencing are High speed and the interaction factor is High speed with low feed . This shows that the Surface Roughness is low at the maximum speed and at minimum feed and with perfect nano material added coolant. Depth of cut does not have much influence over Surface Roughness. From RSM method in Design Expert 8.0 7.1 software came to know the equation to calculate Ra, Ry, Rz and the correct best setting to get good surface finish.

\section{References}

[1]. H Aouici, M A Yallese, A Belbah, Mfameuri and M Elbah Sadhana- Vol. 38, Part 3, June 2013, pp. 429-445._c Indian Academy of Sciences Experimental investigation of cutting parameters influence on surface roughness and cutting forces in hard turning of X38CrMoV5-1 with CBN tool.

[2]. Vikas B. Magdum, Vinayak R. Naik in International Journal of Engineering Trends and Technology (IJETT) - Volume4Issue5May 2013ISSN: 2231-5381 http://www.ijettjournal.org Page 1564Evaluation and Optimization of Machining Parameter for turning of EN 8 steel

[3]. Sudhansu Ranjan Das, Amaresh Kumar, and Debabrata Dhupal in . Effect of Machining Parameters on Surface Roughness in Machining of Hardened AISI 4340 Steel Using Coated Carbide Inserts International Journal of Innovation and Applied Studies ISSN 2028-9324 Vol. 2 No. 4 Apr. 2013, pp. 445-453 (C) 2013 Innovative Space of Scientific Research Journals http://www.issrjournals.org/ijias/Corresponding Author: Sudhansu Ranjan Das .

[4]. P.Subhash, Chandra Bose \& C S P Rao in Evaluation of Optimum Cutting Parameters in Turning of NIMONIC 75 using RSM ISSN : $2319-3182$, Volume -2, Issue - 2, 2013.

[5]. Rodrigues L.L.R.1, Kantharaj A.N.1, Kantharaj B.2, Freitas W. R. C.2 and Murthy B.R.N.1 Research Journal of Recent Sciences ISSN 2277-2502Vol. 1(10), 19-26, October (2012) Effect of Cutting Parameters on Surface Roughness and Cutting Force in Turning Mild Steel.

[6]. Osarenmwinda, J O Empirical model for estimating the surface roughness of machined components under various cutting speed in J. Appl. Sci. Environ. Manage. March, 2012Vol. 16 (1) 65 - 68.

[7]. M. N. Islam, Member, IAENG and Brian Boswell, Proceedings of the World Congress on Engineering 2011 Vol I, WCE 2011, July 6 - 8, 2011, London, U.K.An Investigation of Surface Finish in Dry Turning

[8]. Z. Jurkovic, G . Cukor, I. Andrejcak ISSN 1330-3651 Technical Gazette 17, 4(2010), 397-402 UDC/UDK 621.914.015:519.863 Improving the surface roughness at longitudinal turning using the different optimization methods

[9]. Prof.A.V.N.L.Sharma, Mr.K.V.G.Rama Seshu, Mr. A.Gopichand, 4Dr.K.V.Subbaiah Toolwear and surface finish investigation of hard turning using tool imaging Issue: 3339 - 342.(10) Apr./June 2008 Journal of the Brazilian Society of Mechanical Sciences and Engineering.

[10]. RevuruRevuruSrikant, DameraNageswaraRao, Chalamalasetti Srinivasa Rao, Mendu Siva,Subrahmanyam (Apr./June 2008) Journal of the Brazilian Society of Mechanical Sciences and Engineering Print version ISSN 1678-5878J. Braz. Soc. Mech. Sci. \& Eng. vol.30 no.2 Rio de Janeiro Apr./June 2008 Mathematical Modeling of the Influence of Emulsifier Content on Performance of Cutting Fluids. 